\section{Gasto em atenção primária à saúde em dois governos do Chile pós-ditadura}

\author{
Spending on primary healthcare in two \\ government administrations in Chile since the \\ dictatorship
}

\section{Gasto en atención primaria en salud en dos gobiernos del Chile posdictatorial}

\author{
Fabián Moraga-Cortés 1 \\ Thereza Christina Bahia 1 \\ Clara Aleida Prada 1
}

\section{Resumo}

Desde a Declaração de Alma-Ata, em 1978, a atenção primária à saúde (APS) é considerada componente essencial dos sistemas de saúde. No caso chileno, a gestão da atenção primária foi municipalizada durante a ditadura e mantida pelos governos posteriores, com algumas reformas. O objetivo deste trabalho foi estimar e analisar o gasto em APS no Chile, durante os governos de Sebastián Piñera e Michelle Bachelet. A coleta dos dados financeiros foi orientada pelo Modelo de Contas Nacionais em Saúde (CNS) e, posteriormente, os valores foram deflacionados segundo o Índice de Preços do Consumidor (IPC). A principal fonte das informações foi o Sistema Nacional de Informação Municipal (SINIM). Os resultados mostram que no período houve aumento permanente do gasto em APS, entretanto, a média de variação percentual foi um pouco maior no primeiro governo do que no segundo. A porcentagem do gasto em APS em relação ao gasto público com saúde foi de 21,4\% para os oito anos, tendo poucas variações. Indicadores mostram que a desigualdade entre as regiões administrativas e de saúde está ampliando progressivamente. Por tanto, os repasses destinados a financiar os serviços oferecidos na atenção primária, se bem que crescentes, possivelmente estão sendo mal distribuídos. Isso, junto com outros problemas, como a mercantilização dos serviços e a desintegração da rede, prejudicam a consolidação da APS, sobretudo tratando-se de um sistema de saúde baseado em seguros contributivos como o chileno.

Financiamento da Assistência à Saúde; Atenção à Primária à Saúde; Gastos em Saúde

\author{
Correspondência \\ F. Moraga-Cortés \\ Universidade Estadual de Feira de Santana. \\ Av. Transnordestina s/n, Feira de Santana, BA 44036-900, \\ Brasil. \\ moraga1989@hotmail.com \\ 1 Universidade Estadual de Feira de Santana, Feira de Santana, \\ Brasil.
}




\section{Introdução}

Desde a Conferência de Alma-Ata (Cazaquistão, 1978), a atenção primária à saúde (APS) é considerada a função principal e núcleo fundamental dos sistemas nacionais de saúde, inseparável do desenvolvimento econômico e social, espaço privilegiado para abordar os Determinantes Sociais da Saúde (DSS) e para promover a participação social, procurando diminuir as desigualdades sociais 1 . Posteriormente, várias declarações de órgãos internacionais reafirmaram sua importância para o melhoramento da saúde das populações 2,3,4,5. Pesquisas empíricas têm descrito amplamente as vantagens da APS, independentemente do nível de renda dos países 6,7,8,9.

As experiências de alguns países na região das Américas também mostram os benefícios de políticas orientadas pela APS, tais como a queda da mortalidade infantil, a redução das hospitalizações sensíveis à atenção primária, a menor utilização desnecessária das salas de emergência, dentre outros 10,11,12,13,14,15,16. Entretanto, tentativas para fortalecer a APS foram obstaculizadas pela herança de uma proteção social à saúde fortemente segmentada 17,18,19, apontando para uma vinculação entre sistemas de saúde baseados em seguros, públicos ou privados, com populações e cestas de serviços restritas e uma APS apenas seletiva 20.

O Chile conta com um sistema que tem sido denominado de caráter dual e financiado por seguros contributivos. Existe um subsetor público que outorga atenção de saúde às pessoas filiadas ao Fundo Nacional de Saúde (FONASA - Fondo Nacional de Salud) que, sendo majoritário, cobre os segmentos de mais baixa renda. Por outro lado, existe um conjunto de seguros privados com fins lucrativos denominado Instituciones de Salud Previsional (ISAPRE), que fornece cobertura às camadas mais favorecidas economicamente e com demandas de menor risco aos serviços. O subsetor público é financiado pelas contribuições sociais sobre a renda salarial, o aporte fiscal e os copagamentos dos próprios filiados; o subsetor privado recebe fundos volumosos dos salários e dos copagamentos. A provisão dos serviços, no caso dos filiados ao FONASA, se dá por meio da rede pública de hospitais e centros de atenção primária; as ISAPRE têm convênios com clínicas privadas e vários tipos de profissionais. Ainda assim, existem alguns convênios para a atenção dos assegurados pelo FONASA com prestadores privados. Os planos oferecidos pelas ISAPRE têm, principalmente, serviços curativos não orientados pela APS.

A última reforma implantada no Chile foi denominada Acceso Universal con Garantías Explícitas en Salud (AUGE) e, posteriormente, nomeada como Garantías Explícitas de Salud (GES). Essa reforma implantou a idéia de priorização dos serviços de saúde, ao criar um conjunto de atenções que tem a obrigatoriedade de outorgar-se em prazos estabelecidos, tanto para os filiados ao seguro público quanto para as ISAPRE. Considerando-se o ano de 2020, são 80 os problemas de saúde "garantidos". Algumas consequências negativas da reforma têm sido a desintegração da rede ao diferenciar entre patologias "auge" e "não auge", a maior privatização dos serviços, pois o setor público está compelido a comprar os serviços quando os prazos são vulnerados, e relegação de todas as demais patologias existentes.

Quanto à APS, o país desenvolveu, na primeira década dos anos 2000, o Modelo de Atención Integral de Salud Familiar e Comunitária (MAISFC), orientado por três princípios: atenção centrada no usuário; integralidade da atenção; e continuidade do cuidado, além de nove eixos que incorporam o conteúdo de Alma-Ata 21,22. O novo modelo teve como antecedente a reconversão das unidades de saúde tradicionais em centro de saúde familiar (CESFAM - centros de salud familiar), desde 1997, conformando equipes multiprofissionais com a população a cargo e prioridade nas ações preventivas 23 .

Instrumentos avaliativos reportaram um cumprimento de 56\% dos objetivos da APS em nível nacional, com piores desempenhos dos municípios pequenos, com população rural e maior porcentagem de pobreza 24. Existe ainda discordância entre o novo modelo de APS, que tem foco na prevenção e promoção, e a reforma AUGE-GES, pois esta última é centrada na resolução de um grupo limitado de doenças 25 .

Também persistem importantes dificuldades relacionadas ao processo de municipalização implementado durante a ditadura, tais como a desintegração da rede de atenção, o alto gasto administrativo, a persistência de iniquidades entre municípios pobres e ricos, e a precariedade da infraestrutura física e dos equipamentos das unidades 26,27,28.

A modalidade de financiamento da APS considera o nível central de governo como a principal fonte dos recursos que são destinados para financiar os serviços, entretanto, os municípios podem 
fazer aportes financeiros 17. As transferências desde o nível nacional aos órgãos subnacionais são feitas, princialmente, por meio de um cálculo per capita da população registrada nas unidades, que é corrigido por proxies de necessidades de saúde. Esse mecanismo está regulamentado por lei nacional. Também existem outras vias de repasses que têm financiamentos variáveis segundo o cumprimiento de indicadores 18. Por outro lado, a previsão dos serviços geralmente é feita por órgãos infranacionais (regiões de saúde e municípios) 17.

Tal como é apontado por Coelho 29 , esclarecer os processos de captação e gasto dos recursos constitui uma peça indispensável para que o planejamento e a gestão sejam conduzidos por uma racionalidade sanitária destinada primordialmente a "produzir saúde". É inegável que a discussão em torno da distribuição dos recursos financeiros não é apenas uma questão técnica, pois é atravessada pelo debate entre o paradigma liberal e o social, cada um deles inspirado em concepções conflitantes acerca da função do Estado 30. Mais especificamente, colocar o financiamento dos serviços de saúde como objeto de estudo é relevante para conhecer a prioridade das políticas que são anunciadas pelo Estado.

Recentemente, em 2018, a Organização Pan-Americana da Saúde (OPAS) sugeriu que os países tenham um gasto em atenção primária de, pelo menos, $30 \%$ dos recursos disponíveis para saúde 31 . Não obstante, ainda não há evidência contundente que permita concluir que os países estejam encaminhando seus esforços nessa direção.

Tendo em vista os aportes trazidos pela literatura, este estudo objetivou analisar o gasto em APS, no Chile, entre 2010 e 2017, sendo um caso protótipo de sistema de seguros contributivos. A pesquisa buscou dimensionar as despesas destinadas a financiar os serviços de atenção primária fornecidos pelos municípios e pelas regiões de saúde, avaliando sua variação temporal. O estudo faz o recorte e compara duas conjunturas, que correspondem ao primeiro governo do ex-presidente Sebastián Piñera (2010-2013) e ao segundo governo da ex-presidente Michelle Bachelet (2014-2017).

\section{Metodologia}

É um estudo de caso quantitativo, descritivo-analítico e retrospectivo. A coleta dos dados financeiros foi orientada pelo modelo das Contas Nacionais em Saúde (CNS - National Health Accounts), que adota os princípios do Sistema de Contas de Saúde (System of Health Accounts - SHA) e a classificação do Classificação Internacional para Contas de Saúde (International Classification for Health Accounts - ICHA). Esse modelo, já usado pelo governo chileno para a construção das suas contas, distingue o fluxo dos recursos de uma fonte (origem) para uma entidade (uso) intermediária ou finalista, com a utilização de tabelas 32 . O estudo empregou a primeira tabela, que mostra o fluxo dos recursos das fontes para os agentes intermediários (gestores). Distinguiram-se as fontes entre nacionais e municipais, e os agentes corresponderam aos municípios e às regiões de saúde.

A principal fonte das informações financeiras foi o Sistema Nacional de Informação Municipal (SINIM), plataforma que dispõe de dados sobre as diferentes funções desenvolvidas pelos municípios. No período, houve quatro municípios que não declararam os gastos ao SINIM. Esses dados foram obtidos das contas públicas que os prefeitos apresentaram à comunidade ou estimadas segundo a variação porcentual do período.

No caso dos recursos repassados para as regiões de saúde que não declaram seus gastos ao SINIM, a informação do gasto em atenção primária foi obtida das leis de orçamentos do governo nacional, para cada ano. Esse gasto não está desagregado por município, por este motivo a desagregação do gasto em APS somente pôde ser analisada até as regiões de saúde.

A soma dos gastos geridos pelos municípios e pelas regiões de saúde conformam o gasto em APS. Para dimensioná-lo, foram usados o gasto total com saúde e o gasto público com saúde; este último considera as contribuições dos filiados ao seguro público e o aporte financeiro do Estado. Os dados do ano 2017 ainda não estavam disponibilizados, por este motivo foram estimados tendo como base o valor do ano 2016 acrescido da média de inflação do período.

O gasto em APS também foi relacionado ao gasto geral do governo, ao gasto dos governos municipais e ao Produto Interno Bruto (PIB). Os dois primeiros são a somatória das despesas de todos os setores, além da saúde. Entende-se que o gasto do governo municipal está contido no gasto geral do governo 33. As fontes dessas informações foram o Departamento de Economia da Saúde (DESAL) 
do Ministerio da Saúde, a Escritório de Orçamento do Chile (Dirección de Presupuestos de Chile DIPRES) e o Banco Central do Chile.

O cálculo do gasto em APS per capita considerou três tipos de população superpostas: (1) população total; (2) população filiada ao seguro público de saúde (FONASA); e (3) população registrada nos estabelecimentos de atenção primária municipal. A primeira corresponde aos habitantes totais de determinado município ou região; a segunda, às pessoas cobertas pelo setor público de saúde, sem plano privado; a terceira, às pessoas inscritas anualmente nos estabelecimentos de APS sob gestão municipal. Essa última foi empregada para calcular os repasses aos municípios.

As fontes para determinar os três tipos de populações foram, respectivamente: o Instituto Nacional de Estatísticas (Instituto Nacional de Estadísticas - INE), o FONASA e o SINIM.

A análise do gasto em APS foi feita para regiões de saúde e regiões administrativas. Essas últimas fazem parte da divisão político-administrativa do poder executivo e são lideradas por um intendente regional que atua como representante do Presidente da República sob o território. Em algumas das regiões administrativas mais populosas existem várias regiões de saúde. Nos anos do estudo, havia 16 regiões administrativas e 29 regiões de saúde. As regiões de saúde são encarregadas pela gestão em rede dos estabelecimentos públicos, incluindo os serviços de atenção primária geridos pelos municípios.

A análise dos dados financeiros considerou a deflação dos valores segundo o Índice de Preços ao Consumidor (IPC), com valores referentes ao ano inicial da série considerada (2010) para anular o efeito de desvalorização do dinheiro. Para uma comparação válida, os seguintes indicadores também foram deflacionados segundo o IPC: PIB, gasto geral do governo, gasto dos governos municipais, gasto total com saúde, gasto público com saúde.

Nas tabelas, os dados foram expressos em pesos chilenos (CLP). No texto, também serão especificados alguns valores de maior interesse comparativo, em dólares americanos (USD). Para uma melhor compreensão, o gasto em APS será representado em trilhões, bilhões e milhões de pesos; as cifras per capita apenas em pesos chilenos. A análise considerou a média anual de variação porcentual. Como indicador de desigualdade, consideraram-se as diferenças entre valores mínimos e máximos e as diferenças das médias entre os decis 1 e 10.

\section{Resultados}

O gasto aumentou, em valores nominais, de CLP 702 bilhões (880 milhões de dólares, em câmbio do dia 26 de novembro de 2019), em 2010, para CLP 1,8 trilhão, em 2017, implicando um aumento de 2,5 vezes (Figura 1). Em valores deflacionados, o incremento foi de 2 vezes, passando de CLP 702 bilhões, no início do período, para CLP 1,4 trilhão. Essa última mudança também pode ser expressa em um incremento porcentual de $102 \%$. O gasto em atenção primária acumulado nos oito anos de estudo, em valores deflacionados, ficou em torno de CLP 8,3 trilhões. Desse montante de recursos, 60\% (CLP 5,0 trilhões) foram gastos durante o governo de Michelle Bachelet e 40\% (CLP 3,3 trilhões) no governo de Sebastián Piñera.

Considerando apenas os valores deflacionados, a taxa média de variação foi de 10,6\% ao ano. O ritmo de crescimento foi maior no período do governo de Sebastián Piñera, registrando uma variação de $11,6 \%$ ao ano. Entre 2010 e 2011, as despesas cresceram 9,4\%; entre 2011 e 2012, houve o maior incremento do período analisado, de 14,7\%; e entre 2012 e 2013, a taxa foi de 10,8\%. Destaca-se que, por se localizar ao início da série, o cálculo da variação durante o governo de Sebastián Piñera foi baseado em três medições, sendo o ano de 2010 o momento inicial dos registros.

Durante o governo de Michelle Bachelet, a média de variação foi menor, situando-se em 9,8\% ao ano. Entre 2013 e 2014, o ritmo de variação continuou com a tendência dos anos anteriores, ficando em 10,7\%. Entretanto, entre 2014 e 2015, registrou-se o incremento mais baixo do período, de 8,4\%. Nos anos seguintes, a variação foi maior, mas não conseguiu se aproximar do nível máximo observado no pico do primeiro governo. Entre 2015 e 2016, o aumento foi de 10,5\% e, entre 2016 e 2017, ficou em $9,5 \%$.

A composição do gasto para o período 2010-2017, segundo fontes e agentes, é mostrada na Tabela 1. As fontes nacionais foram majoritárias, sendo quantificadas em CLP 7,4 trilhões nos oito anos, o que 


\section{Figura 1}

Evolução do gasto em atenção primária à saúde (APS). Chile, 2010 a 2017.

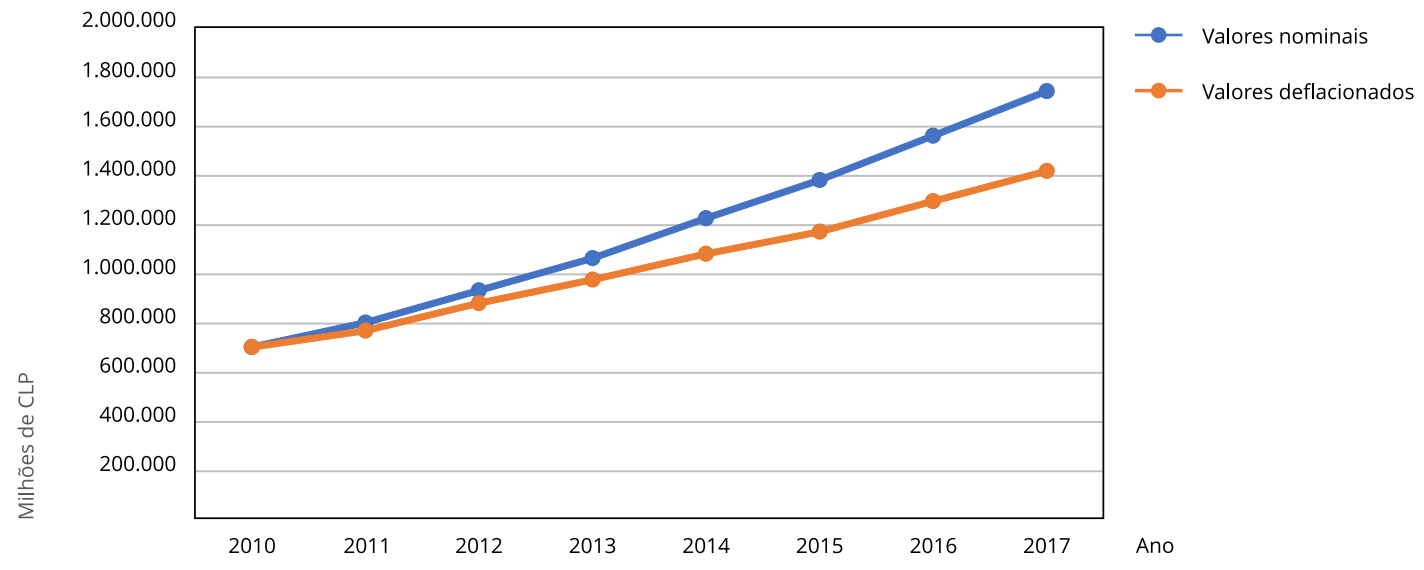

\section{Tabela 1}

Composição do gastos em atenção primaria à saúde (APS) segundo fontes e agentes em valores deflacionados. Chile, 2010 a 2017 (milhões de CLP).

\begin{tabular}{|c|c|c|c|c|c|c|c|c|}
\hline \multirow[t]{3}{*}{ Anos } & \multicolumn{4}{|c|}{ Fontes (milhões de CLP) } & \multicolumn{4}{|c|}{ Agentes (milhões de CLP) } \\
\hline & \multicolumn{2}{|c|}{ Nacionais } & \multicolumn{2}{|c|}{ Municipais } & \multicolumn{2}{|c|}{ Municípios } & \multicolumn{2}{|c|}{ Regiões de saúde } \\
\hline & Gastos em APS & $\%$ & Gastos em APS & $\%$ & Gastos em APS & $\%$ & Gastos em APS & $\%$ \\
\hline 2010 & 627.128 & 89,3 & 74.814 & 10,7 & 624.238 & 88,9 & 77.704 & 11,1 \\
\hline 2011 & 685.245 & 89,3 & 82.391 & 10,7 & 690.204 & 89,9 & 77.431 & 10,1 \\
\hline 2012 & 786.880 & 89,4 & 93.552 & 10,6 & 774.071 & 87,9 & 106.362 & 12,1 \\
\hline 2013 & 872.630 & 89,4 & 103.198 & 10,6 & 856.637 & 87,8 & 119.191 & 12,2 \\
\hline 2014 & 961.407 & 89,0 & 119.001 & 11,0 & 953.703 & 88,3 & 126.705 & 11,7 \\
\hline 2015 & 1.009 .735 & 86,2 & 161.780 & 13,8 & 1.049 .341 & 89,6 & 122.175 & 10,4 \\
\hline 2016 & 1.142 .533 & 88,2 & 152.530 & 11,8 & 1.150 .627 & 88,8 & 144.435 & 11,2 \\
\hline 2017 & 1.271 .200 & 89,6 & 146.956 & 10,4 & 1.265 .725 & 89,3 & 152.431 & 10,7 \\
\hline Total & 7.356 .758 & 88,7 & 934.222 & 11,3 & 7.364 .546 & 88,8 & 926.434 & 11,2 \\
\hline
\end{tabular}

Fonte: elaboração própria com base no Sistema Nacional de Informação Municipal (SINIM - http://datos.sinim.gov.cl/).

equivale a 88,7\% do total. As fontes municipais forneceram CLP 0,9 trilhão, representando 11,3\% do gasto com a atenção primária. Os municípios foram os principais agentes, dado que geriram a maior parte das despesas - CLP 7,4 trilhões - representando 88,8\%; e as regiões de saúde geriram CLP 0,9 trilhão, representando $11,2 \%$ do total.

Cabe destacar que, o incremento do gasto em atenção primária também é observado em termos per capita. A Figura 2 mostra a evolução do gasto em APS per capita segundo população total, população filiada ao FONASA e população registrada nos estabelecimentos de atenção primária. A evidência mostra que o gasto em APS per capita segundo a população total é menor ao ser comparado com o gasto per capita baseado no segmento que é filiado ao FONASA e o que é registrado, dado que as duas últimas formam um subconjunto da primeira. Em 2017, o gasto em APS per capita calculado segundo a população total foi $66 \%$ do gasto em APS per capita baseado no segmento registrado nas unidades de 
Figura 2

Gasto em atenção primária à saúde (APS) per capita segundo valores deflacionados. Chile, 2010 a 2017.

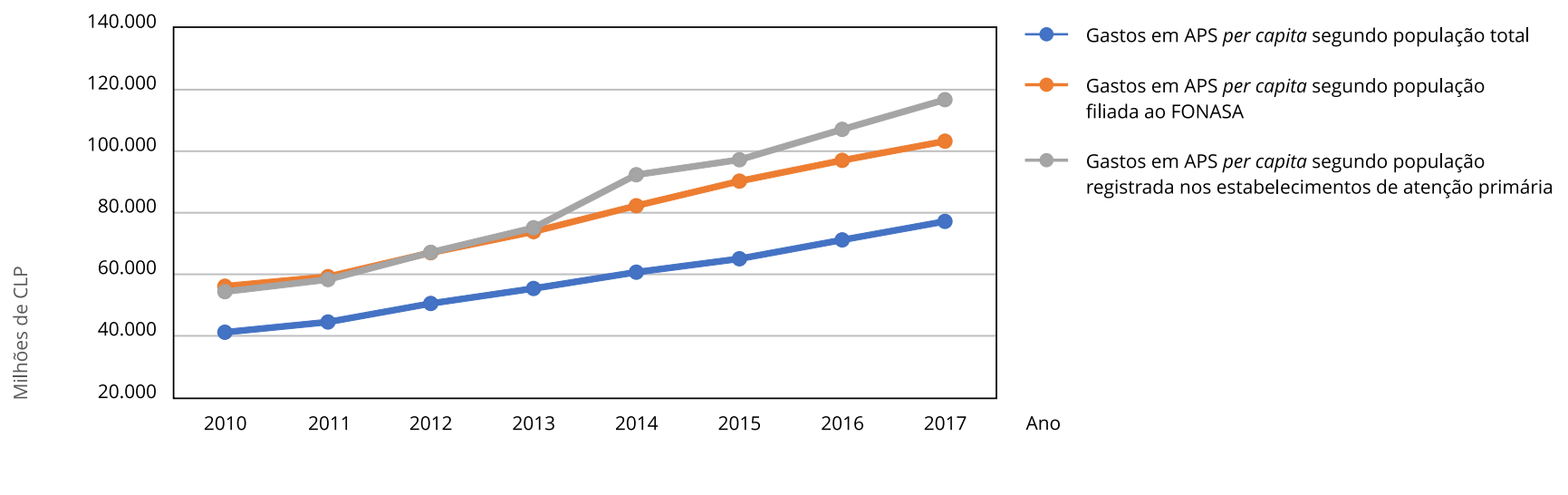

FONASA: Fundo Nacional de Saúde.

atenção primária municipal. Tal como foi apontado na metodologia, essa última constitui a base para uma das transferências do Nível Central aos municípios, por meio do estabelecimento de um valor per capita que é ajustado por alguns proxies de necessidades de saúde.

A Tabela 2 mostra a porcentagem do gasto calculado sobre PIB, gasto geral do governo, gasto total com saúde, gasto público com saúde e gasto dos governos municipais. As despesas representaram 3,3\% do gasto geral do governo para todo o período, passando de 2,7\%, em 2010, para 3,8\%, em 2017. O gasto também foi quantificado em 10,4\% do gasto total com saúde, o que mostra um acréscimo de 9,3\%, em 2010, para 10,9\%, em 2017. O gasto como proporção do gasto público com saúde apresenta o menor aumento dentre os quatro indicadores: passou de 19,7\%, em 2010, para 21,5\%, em 2017, tendo ficado em 21,4\% para todo o período. A média de variação desse indicador foi apenas de $1 \%$ ao ano, tendo dois momentos em que foi registrada uma leve diminuição: entre 2014 e 2015, quando representou $22,1 \%$ e $21,7 \%$, respectivamente; e entre 2016 e 2017 , quando passou de $21,8 \%$ para $21,5 \%$. Por outro lado, o gasto foi estimado em $24,6 \%$ do gasto dos governos municipais para os oito anos. Registrou-se um aumento, passando de 21,2\% em 2010 para 26,3\% em 2017. O maior incremento foi observado entre 2010 e 2011, com 21,2\% e 26,3\%, respectivamente. A média de variação para os oito anos foi de $3 \%$ ao ano. Os recursos para a atenção primária registraram uma maior proporção no nível municipal do que no nível nacional, no período analisado.

A Tabela 3 apresenta os indicadores de desigualdade dos recursos financeiros para regiões de saúde e administrativas. Em 2010, o gasto per capita da região de saúde com o maior gasto representou 2,5 vezes os fundos destinados à região com o menor gasto, ao tempo em que a relação entre o decil 1 e o 10 foi de 1,9 vez. O primeiro indicador aumentou progressivamente, até alcançar, em 2017, 3,6 vezes. A relação entre os decis, mesmo aumentando progressivamente, apresentou um avanço mais leve; em 2017, 10\% das regiões de saúde com as maiores despesas registraram um gasto per capita que representou 2,3 vezes os recursos repassados às regiões de saúde com menor gasto.

Ao analisar o gasto desagregado segundo regiões administrativas, os dados mostram diferenças pouco significativas entre as localidades de menor e maior gastos per capita, e menores mudanças ao comparar as cifras, entre 2010 e 2017. No último ano, a região administrativa com as maiores despesas per capita apresentou recursos que foram 1,9 vez os destinados à região administrativa com o pior gasto per capita, ao tempo em que $10 \%$ das regiões com maior gasto per capita registraram repasses que representaram 1,7 vez os repassados às regiões com os menores recursos financeiros per capita.

Os dois indicadores empregados mostram, entre 2010 e 2017, uma ampliação das diferenças para as regiões administrativas com melhor e pior despesas per capita. Tal comprovação sugere que a alocação de recursos está sendo cada vez mais desigual. Por outro lado, as diferenças de gasto tendem a 
Tabela 2

Proporção do gasto em atenção primária à saúde (APS) sobre o gasto geral do governo, o gasto total em saúde, o gasto público em saúde e o gasto dos governos municipais. Chile, 2010 a 2017.

\begin{tabular}{|c|c|c|c|c|c|}
\hline \multirow[t]{2}{*}{ Anos } & \multicolumn{4}{|c|}{ Nível nacional } & \multirow{2}{*}{$\begin{array}{l}\text { Nível municipal } \\
\text { Gasto em APS/gasto dos } \\
\text { governos municipais (\%) }\end{array}$} \\
\hline & $\begin{array}{c}\text { Gasto público em } \\
\text { saúde/PIB (\%) }\end{array}$ & $\begin{array}{l}\text { Gasto em APS/gasto } \\
\text { geral do governo (\%) }\end{array}$ & $\begin{array}{l}\text { Gasto em APS/gasto } \\
\text { total em saúde (\%) }\end{array}$ & $\begin{array}{l}\text { Gasto em APS/gasto } \\
\text { público em saúde (\%) }\end{array}$ & \\
\hline 2010 & 0,6 & 2,7 & 9,3 & 19,7 & 21,2 \\
\hline 2011 & 0,7 & 2,9 & 9,7 & 20,6 & 23,6 \\
\hline 2012 & 0,7 & 3,1 & 10,2 & 21,3 & 24,1 \\
\hline 2013 & 0,8 & 3,3 & 10,4 & 21,7 & 24,9 \\
\hline 2014 & 0,8 & 3,5 & 10,6 & 22,1 & 25,0 \\
\hline 2015 & 0,9 & 3,5 & 10,5 & 21,7 & 24,8 \\
\hline 2016 & 0,9 & 3,6 & 10,9 & 21,8 & 25,0 \\
\hline 2017 & 1,0 & 3,8 & 10,9 & 21,5 & 26,3 \\
\hline Total & 0,8 & 3,3 & 10,4 & 21,4 & 24,6 \\
\hline
\end{tabular}

Fonte: elaboração própria com base no Sistema Nacional de Informacão Municipal (SINIM - http://datos.sinim.gov.cl/), Direção de Orçamentos (DIPRES - https://www.dipres.gob.cl/598/w3-propertyvalue-15407.html) e Ministério da Saúde (MINSAL - http://ies.minsal.cl/gastos/sha2011/indicadores/1).

Tabela 3

Indicadores de desigualdade do gasto em atenção primária à saúde (APS) per capita para regiões administrativas e de saúde. Chile, 2010 a 2017.

\begin{tabular}{lcccc}
\hline Anos & \multicolumn{2}{c}{$\begin{array}{c}\text { Regiões de saúde } \\
\text { Quociente mínimo/ } \\
\text { máximo }\end{array}$} & $\begin{array}{c}\text { Regióes administrativas } \\
\text { Quociente d10/d1 }\end{array}$ \\
\hline 2010 & 2,5 & 1,9 & 1,6 & 1,5 \\
2011 & 2,7 & 1,9 & 1,7 & 1,5 \\
2012 & 2,7 & 2,0 & 1,7 & 1,5 \\
2013 & 3,2 & 2,1 & 1,8 & 1,6 \\
2014 & 3,2 & 2,1 & 1,7 & 1,6 \\
2015 & 3,3 & 2,2 & 1,7 & 1,6 \\
2016 & 3,2 & 2,1 & 1,6 & 1,6 \\
2017 & 3,6 & 2,3 & 1,9 & 1,7 \\
\hline
\end{tabular}

Fonte: elaboração própria com base no Sistema Nacional de Informação Municipal (SINIM - http://datos.sinim.gov.cl/). 
aumentar quando os repasses são desagregados para unidades de análises menores, por exemplo, em 2017, quando a análise considera regiões administrativas, a relação entre valores máximos e mínimos foi de 1,9 vez, mas subiu para 3,6 vezes se os repasses são diferenciados no nível das regiões de saúde.

\section{Discussão}

Este estudo analisou o gasto em APS em dois governos chilenos após a ditadura: o primeiro governo do ex-presidente Sebastián Piñera (2010-2013), conformado por uma coalisão de partidos políticos de centro-direita; e o segundo governo da ex-presidente Michelle Bachelet (2014-2017), de orientação centro-esquerda. Chama a atenção a diferença no ritmo de gasto, dado que o governo de direita aumentou o gasto em APS mais do que o governo de esquerda. Entretanto, é necessário entender que a curva de crescimento do financiamento setorial impõe, por sua própria dinâmica, restrições de crescimento, pois a tendência da taxa é de desaceleração após ser atingido um certo ponto.

Por outro lado, a sociedade chilena pós-ditadura caracteriza-se pela despolitização da vida pública e pela massificação do consumo baseado no crédito como meio (medium) de integração social 34. Sendo assim, a discussão em torno dos fins sociais passou a ser ditada pelo discurso econômico hegemônico, de clara orientação neoliberal e tecnocrático. Nesse contexto, as categorias de esquerda e direita, entendidas da forma tradicional, deixaram de ser um fator diferenciador das políticas sociais.

$\mathrm{O}$ incremento do GAPS, em valores nominais, quantificado neste estudo (2,5 vezes) foi menor que os achados de Debrot \& Ibáñez 35, que observaram um incremento de 3,3 vezes, considerando os valores nominais entre 2003 e 2013. É um período diferente do analisado neste trabalho, porém, a comparação com um período mais amplo mostra que o incremento de 2,5 vezes foi menor do que o observado em anos prévios. A DIPRES, que é a instituição encarregada pela programação financeira do governo nacional, quantificou a evolução do valor per capita empregado para calcular os repasses aos municípios, estabelecendo 5\% de incremento entre 1997 e 2004. Entretanto, essa cifra aumentou para 9\% durante a discussão da reforma AUGE-GES, aprovada em 2005 36. Possivelmente, já passados quase 20 anos da aprovação da reforma, o ritmo de crescimento diminuiu, o que explica o menor incremento estimado neste estudo.

A variação de $10 \%$ ao ano observada nesta pesquisa foi menor do que a cifra informada pela DIPRES (14\%) 36. Esse cálculo feito pela instituição de governo abrangeu o período de 1990 até 2012, além disto, não incluiu os aportes vindos das fontes municipais. Apesar dessas diferenças metodológicas, pode-se afirmar que o ritmo de crescimento evidenciado neste estudo foi menor do que o observado nas décadas de 90 e 2000.

Segundo o mesmo órgão, em 2012 o gasto em APS representou 20\% do gasto público em saúde 36. A cifra calculada neste estudo, para o mesmo ano, foi similar (21\%). A proporção do gasto em APS sobre o gasto total com saúde foi quantificada em 10,4\% para todo o período. Essas cifras mostram a pouca prioridade da APS na distribuição nacional dos recursos e mostra que o país ainda está longe de cumprir a orientação da OPAS para que as nações da região gastem, pelo menos, 30\% dos recursos na APS 31. Esse descumprimento, por seu turno, está na contramão da evidência internacional, que assinala que sistemas com uma atenção primária forte conseguem resolver de $80 \%$ a $85 \%$ dos problemas de saúde neste nível de atenção, convertendo o gasto na APS em uma inversão estratégica dos recursos que as nações dispõem para o setor 37.

Evidentemente, essa contradição entre o nível do gasto em APS e a importância sanitária da APS é apenas um dos fatores que dificultam a sua consolidação. O Chile apresenta os obstáculos que têm sido reportados mundialmente para o desenvolvimento da APS: hospitalocentrismo, fragmentação das ações e mercantilização dos serviços 38,39,40. Por outro lado, a educação médica está excessivamente concentrada na formação de especialistas no âmbito hospitalar e existem resistências dos atores educativos à mudança deste paradigma 41 .

A diferença entre um gasto em APS per capita baseado na população total, menor do que o gasto per capita baseado na população registrada nas unidades de APS, faz parte das distorções acarretadas pelos sistemas de seguros de saúde. Tal forma de cálculo, nomeada como "capitação ponderada", foi proposta para o Brasil pelo governo do presidente Jair Bolsonaro, com a diferença de que o Sistema Único de Saúde (SUS) é universal e regido por leis diferentes daquelas que sustentam o sistema chileno 42. 
Os sistemas de saúde que promovem o livre mercado, dentre eles, o chileno, têm dificuldades maiores para a expansão da APS, dada a composição público-privada da rede de prestadores e a segmentação da população pertencente aos diversos tipos de seguros 43,44. A separação entre prestação e financiamento de serviços de saúde é apontada como um desafio para a construção de redes de serviços de saúde coordenadas pela APS 45. No caso chileno, essa separação está representada por uma rede pública de saúde que tenta expandir políticas de fortalecimento da APS, a exemplo do MAISFC, dentro da lógica de financiamento própria dos seguros de saúde. Isso faz com que o repasse dos recursos financeiros seja estimado segundo a população registrada nos estabelecimentos de atenção primária municipal e não na população residente num determinado município ou região. Ao mesmo tempo, há políticas que permitem o acesso "condicionado" aos serviços de saúde na atenção primária para toda a população, inclusive para pessoas filiadas aos seguros privados, os já referidos ISAPRE. Exemplo dessas políticas é o Programa Nacional de Imunizações, de caráter universal e financiado com fundos públicos 46. Outro caso corresponde às mulheres grávidas e crianças menores de seis anos de idade, pois têm a garantia legal de atenção, independentemente do tipo de asseguramento de saúde que possuam 47 . Esses grupos podem ser atendidos nos dispositivos de atenção primária só cumprindo os requisitos estabelecidos, mas, por não fazerem parte da população registrada nos estabelecimentos de atenção primária municipal, esses serviços não são financiados pelas transferências feitas do nível nacional aos municípios. No caso da população migrante, que geralmente não faz parte da população registrada nos estabelecimentos de atenção primária municipal, acrescentam-se as múltiplas dificuldades administrativas para ofertar serviços a migrantes irregulares sem registro migratório e para o planejamento efetivo das ações de saúde 48.

Além disso, alguns dos serviços preventivos e de promoção da saúde inclusos no MAISFC devem ser fornecidos para toda a população dos territórios. Assim, na prática, a existência da população registrada nos estabelecimentos de atenção primária municipal incentiva a focalização dos serviços apenas na população registrada, pois são estas as pessoas consideradas no cálculo dos repasses. Dessa maneira, as atenções não curativas não são priorizadas pois não têm mecanismo de financiamento que garantam os recursos necessários para a oferta destes serviços.

Consequentemente, as distorções geradas pela diferenciação entre a população total e o subconjunto que é registrado nas unidades de saúde fazem com que os serviços administrados pelos municípios e pelas regiões de saúde forneçam serviços para uma população maior do que a empregada para o cálculo dos repasses financeiros. Essa situação promove o subfinanciamento da atenção primária, dificulta a continuidade do cuidado e a territorialização da rede dos serviços, que são atributos fundamentais dos sistemas de saúde com foco na APS 7.

Por outro lado, chama a atenção a alta proporção do gasto em APS em relação ao gasto dos governos municipais. O Chile caracteriza-se por ser um país com baixa descentralização dos recursos financeiros. Horst 49 estabeleceu que no Chile o gasto dos governos municipais representa $12 \%$ do gasto geral do governo, sendo uma cifra menor em comparação com outros países membros da Organização para a Cooperação e o Desenvolvimento Econômico (OCDE), que têm uma média de 30\%. Sendo assim, o baixo gasto dos governos municipais faz com que a proporção do gasto em APS seja alta, dado que os recursos repassados do nível central para os municípios são contabilizados dentro do gasto dos governos municipais.

É preocupante a constatação de que as diferenças entre as regiões com altos e baixos gastos estão ampliando, gerando dúvidas razoáveis em relação à equidade na distribuição territorial do gasto em APS. Apesar dessa evidência não ser suficiente para afirmar a sua contribuição à consolidação das iniquidades, outorga uma primeira provocação para o seu estudo posterior. Faz-se relevante a realização de estudos que considerem proxies de necessidades de saúde no nível municipal ou regional, para estabelecer se nos locais com menor gasto em APS per capita existem maiores necessidades de saúde ou vice-versa.

Outro trabalho estimou, por meio da mesma metodologia desta pesquisa, o gasto em APS em 36 países de renda média e média baixa, chegando a estabelecer uma variação entre USD 15 e USD 60 per capita, entre os anos 2011 e 2016, a depender do uso das oito classificações consideradas pelos pesquisadores 50. A maioría dos países inclusos nessa pesquisa tem um PIB per capita muito mais baixo do que o chileno, pelo qual o diferente nível de desenvolvimento econômico dificulta uma comparação confiável. 
Este trabalho evidenciou que o ritmo de crescimento, durante o período analisado, foi menor do que os reportes de outras investigações que consideraram décadas anteriores, o que leva à conclusão de que a expansão orçamentária da APS estaria diminuindo. O estudo também achou que o país ainda está longe de atingir o patamar mínimo estabelecido pela OPAS para o gasto em atenção primária. Em outra perspectiva, sugere-se que a alocação do gasto para os diferentes municípios, regiões de saúde e regiões administrativas não está sendo orientada para melhorar o nível de equidade do sistema de saúde.

Essas evidências são ainda mais pertinentes tendo em vista o momento atual do país, marcado pela mobilização da sociedade civil contra a mercantilização dos direitos sociais. De fato, a pressão social tem forçado os setores conservadores no sentido da elaboração de uma nova Constituição. Nesse contexto, organizações sociais da saúde demandaram a criação de um sistema universal de saúde baseado na prevenção e promoção, mediante uma forte expansão orçamentária da APS 51. Entretanto, é preocupante que entre acadêmicos sanitaristas não haja consenso sobre a necessidade de um sistema universal de saúde, conceito que tende a ser misturado com um sistema de seguro único 52,53. A construção de um sistema universal de saúde não tem sido parte das poucas oportunidades de transformação do sistema nos últimos anos 54 . O anterior traz à tona o perigo da confusão das estratégias de luta adequadas para uma APS abrangente, pois uma adequada conceituação sobre o papel da APS nos sistemas universais de saúde é necessária para a mobilização dos atores sociais em prol de uma atenção primária forte, com recursos suficientes e adequados, tal como foi concebida em Alma-Ata.

\section{Conclusão}

Os resultados obtidos são úteis para orientar os decisores do nível nacional e gestores municipais, podendo ser discutidos nas instâncias de planejamento que são organizadas pelas instituições do setor saúde. Além disso, as mesmas conclusões são valiosas para validar as demandas de movimentos sociais no âmbito da saúde, tendentes a consolidar uma efetiva expansão da APS.

Dentre as fortalezas deste estudo, está o fato de basear-se na metodologia das CNS, que permitiu a coleta das informações financeiras distinguindo fontes e agentes. Isso é relevante, na medida em que os relatórios da DIPRES consideram apenas as fontes nacionais. Dentre as limitações, estão as várias dificuldades para estabelecer comparações internacionais em torno do gasto, pois cada país tem arranjos específicos de fontes e agentes.

Finalmente, este estudo comprovou que as diferenças do gasto em APS entre governos são mínimas. Desse ponto de vista, é sugerido para outros trabalhos considerar não apenas as mudanças nas coalizões dos governos, mas também a comparação de políticas que intervenham na APS. No caso chileno, a implantação do MAISFC, ou da reforma AUGE-GES, constitui um bom caso para futuras investigações. 


\section{Colaboradores}

F. Morada-Cortés, T. C. Bahia e C. A. Prada contribuíram na concepção da ideia de pesquisa, elaboração do projeto, coleta e análise dos dados, redação do manuscrito, e aprovação da versão final.

\section{Informações adicionais}

ORCID: Fabián Moraga-Cortés (0000-0001-62075882); Thereza Christina Bahia (0000-0003-47874103); Clara Aleida Prada (0000-0002-4686-2197).

\section{Referências}

1. Organização Mundial da Saúde. Declaração de Alma-Ata. https://www.paho.org/hq/index. php?option $=$ com_docman\&task $=$ doc_view \& gid $=19004 \&$ Itemid $=270 \&$ lang $=$ en $($ acessado em 12/Jan/2018).

2. Organización Mundial de la Salud. Estrategia mundial de salud para todos en el año 2000. https://apps.who.int/iris/hand le/10665/197241 (acessado em 01/Ago/2017).

3. Organização Mundial da Saúde; Organização Pan-Americana da Saúde. Renovação da atenção primária em saúde nas Américas. https:// www.paho.org/bra/index.php?option $=\mathrm{com}_{-}$ docman\&view=download\&category_slug =atencao-primaria-em-saude-944\&alias = 737-renovacao-da-atencao-primaria-em-sau de-nas-americas-7\&Itemid $=965$ (acessado em 14/Jan/2017).

4. Organización Mundial de la Salud. Declaración política de Río sobre determinantes sociales de la salud. https://issuu.com/researchfor health/docs/name9d6374 (acessado em 12/ Ago/2018).

5. Organización Mundial de la Salud. Declaración de Astana sobre atención primaria de salud: desde Alma-Ata hasta la cobertura universal de salud y los Objetivos de Desarrollo Sostenible. https://www.who.int/docs/ default-source/primary-health/declaration/ gcphc-declaration-sp.pdf (acessado em 22/ Dez/2018).

6. Starfield B. Atenção primária e sua relação com a saúde. In: Starfield B, organizador. Atenção primária, equilíbrio entre necessidades de saúde, serviços e tecnologia. Brasília: Organização das Nações Unidas para a Educação, a Ciência e a Cultura; 2002. p. 19-42.

7. Starfield B. Sistemas de atenção primária em nações ocidentais industrializadas. In: Starfield B, organizadora. Atenção primária, equilíbrio entre necessidades de saúde, serviços e tecnologia. Brasília: Organização das Nações Unidas para a Educação, a Ciência e a Cultura; 2002. p. 565-96.

8. Starfield B. Primary care: an increasingly important contributor to effectiveness, equity, and efficiency of health services. Gac Sanit 2012; 16:20-6.

9. Rohde J, Cousens S, Chopra M, Tangcharoensathien V, Black R, Bhutta ZA, et al. 30 years after Alma-Ata: has primary health care worked in countries? Lancet 2008; 372:950-61.

10. Almeida G, Artaza O, Donoso N, Fábrega R. La atención primaria de salud en la Región de las Américas a 40 años de la Declaración de Alma-Ata. Rev Panam Salud Pública 2018; 42:1-6.

11. Aquino R, Oliveira N, Barreto M. Impact of the Family Health Program on infant mortality in Brazilian Municipalities. Am J Public Health 2009; 99:87-93. 
12. Dourado I, Oliveira VB, Aquino R, Bonolo P, Lima-Costa MF, Medina MG, et al. Trends in primary health care-sensitive conditions in Brazil. The role of the Family Health Program (Project ICSAP-Brasil). Med Care 2011; 49:579-84.

13. Hone T, Rosella D, Barreto M, Majeed A, Millett C. Association between expansion of primary healthcare and racial inequalities in mortality amenable to primary care in Brazil: a national longitudinal analysis. PLoS Med 2017; 14:e1002306.

14. Rosella D, Aquino R, Barreto M. Impact of the Family Health Program on the quality of vital information and the reduction of child unattended deaths in Brazil: an ecological longitudinal study. BMC Public Health. 2010; 10:380.

15. Almaguer M, Herrera R, Alonso J, Magrans C, Mañalich R, Martínez A. Primary health care strategies for the prevention of end-stage renal disease in Cuba. Kidney Int Suppl 2005; (97):S4-10.

16. Guttmann A, Lam K, Shipman S, Goodman A. Primary care physician supply and childrens health care use, access, and outcomes: findings from Canada. Pediatrics 2010; 125:1119-26.

17. Giovanella L, Almeida PF, Romero RV, Oliveira S, Tejerina H. Panorama de la Atención Primaria de Salud en Suramérica: concepciones, componentes y desafíos. Saúde Debate 2015; 39:300-22.

18. Giovanella L, Almeida PF. Atenção primária integral e sistemas segmentados de saúde na América do Sul. Cad Saúde Pública 2017; 33 Suppl 2:e00118816.

19. Giovanella L, Romero RV, Tejerina H, Almeida PF, Ferreira G, Goede H, et al. Atención Primaria en Salud en Suramérica: ¿Reorientación hacia el cuidado integral? In: Giovanella L, organizadora. Atención primaria en salud en Suramérica. Rio de Janeiro: Instituto Sul-Americano de Governo em Saúde; 2015. p. 23-58.

20. Labonté R, Sanders D, Baum F, Schaay N, Packer C, Laplante D, et al. Aplicación, efectividad y contexto político de la atención primaria integral de salud: resultados preliminares de una revisión de la literatura mundial. Revista Gerencia y Políticas de Salud 2009; 8:14-29.

21. Ministerio de Salud. Orientaciones para la implementación del modelo de atención integral de salud familiar y comunitaria. http://buenas practicasaps.cl/wp-content/uploads/2013/11/ Orientaciones-para-la-implementacion-delModelo-de-Atenci\%C3\%B3n-Integral-deSalud-Familiar-y-Comunitaria_DIVAP_2013. pdf (acessado em 12/Jan/2018).

22. Dois A, Bravo P, Soto G. Atributos y características de los principios orientadores del Modelo de Atención Integral de Salud Familiar y Comunitária desde la perspectiva de expertos en APS. Rev Med Chile 2017; 145:879-87.

23. Téllez A. Atención primaria: factor clave en la reforma de salud. Santiago: Pontificia Universidad Católica de Chile; 2006; (Temas de la Agenda Pública, 2).
24. García-Huidobro D, Barros X, Quiroz A, Barría $M$, Soto $G$, Vargas $M$, et al. Modelo de atención integral en salud familiar y comunitaria en la atención primaria chilena. Rev Panam Salud Pública 2018; 42:e160.

25. Bass del Campo C. Modelo de salud familiar en Chile y mayor resolutividad de la atención primaria de salud: ¿contradictorios o complementarios? Medwave 2012; 12:e5571.

26. Montoya-Aguilar C. Atención primaria de salud. Alma-Ata una otra vez y la experiencia de Chile. Cuad Méd-Soc (Santiago de Chile) 2008; 48:147-54.

27. Sepúlveda C. El significado histórico de la reforma de la atención de salud en Chile. Cuad Méd-Soc (Santiago de Chile) 2004; 43:21-36,

28. Goic A, Armas R. Descentralización en salud y educación: la experiencia chilena. Rev Med Chile 2003; 131:788-98.

29. Coelho TC. O desafio de planejar e gastar em saúde. In: Coelho TC, Teles AS, Ferreira MPS, organizadores. Financiamento do SUS, abrindo a caixa preta. Feira de Santana: UEFS Editora; 2016. p. 9-12.

30. Globekner OA. A saúde entre o público e o privado, o desafío da alocação social dos recursos sanitários escassos. Curitiba: Jurúa Editora; 2011.

31. Organización Panamericana de la Salud. "Salud Universal en el Siglo XXI: 40 años de Alma-Ata". Informe de la Comisión de Alto Nivel. http://iris.paho.org/xmlui/hand le/123456789/50960 (acessado em 19/ Out/2019).

32. Parners for Health Reformsplus. Understanding national health accounts: the metodology and implementation process. Bethesa: Primer for Policymakers; 2003.

33. Dirección de Presupuestos, Ministerio de Hacienda. Estadísticas de las finanzas públicas 2008-2017. http://www.dipres.gob.cl/598/ articles-174525_doc_pdf.pdf (acessado em/ Out/2019).

34. Moullian T. Chile actual, anatomía de un mito. Santiago de Chile: LOM Ediciones; 2002. (Colección Escafandra).

35. Debrot D, Ibáñez C. Análisis y propuesta de financiamiento de la atención primaria de salud municipal. https://www.achm.cl/index. $\mathrm{php/documentos/file/514-analisis-y-pro}$ puesta-de-financiamiento-de-la-atencion-pri maria-de-salud-municipal (acessado em 03/ Set/2018).

36. Dirección de Presupuestos, Ministerio de Hacienda. Sistema público de salud. Situación actual y proyecciones fiscales. https://www.di pres.gob.cl/598/articles-117505_doc_pdf.pdf (acessado em 12/Set/2018).

37. Giraldo A, Vélez C. La atención primaria de salud: desafíos para su implementación en América Latina. Aten Prim (Barc, Ed impr) 2013; 45:384-92. 
38. Organização Mundial da Saúde. Relatório Mundial de Saúde 2008. Cuidados de Saúde primários: agora mais que nunca. https://www. who.int/eportuguese/publications/whr08_ pr.pdf (acessado em 12/set/2018).

39. Rosas A, Narciso V, Cuba M. Atributos de la atención primaria de salud (APS): una visión desde la medicina familiar. Acta Méd Peru 2013; 30:42-7.

40. Centro de Políticas Públicas UC, Pontifícia Universidad Católica de Chile. Fortalecimiento de la atención primaria de salud: propuestas para mejorar el sistema chileno. https://politicaspublicas.uc.cl/wp-content/ uploads/2015/02/fortalecimiento-de-laatencion-primaria-de-salud-propuestas-paramejorar-el-sistema-sanitario.pdf (acessado em 12/Set/2018).

41. Parada-Lezcano M, Romer MI, Moraga F. Educación Médica para la Atención Primaria de Salud: visión de los docentes y estudiantes. Rev Med Chile 2016; 144:1059-66.

42. Ministério da Saúde. Portaria no 2.979 , de 12 de novembro de 2019. Institui o Programa Previne Brasil, que estabelece novo modelo de financiamento de custeio da Atenção Primária à Saúde no âmbito do Sistema Único de Saúde, por meio da alteração da Portaria de Consolidação no 6/GM/MS, de 28 de setembro de 2017. Diário Oficial da União 2019; 13 nov.

43. Organização Pan-Americana da Saúde. Redes integradas de serviços de saúde. Conceitos, opções de políticas, e folha de rota para sua implementação nas américa. https://www.paho. org/uru/index.php?option=com_docman\& view $=$ download \&alias $=145$-redes-integradasde-servicios-de-salud-aps-n4\&category_slu $\mathrm{g}=$ publicaciones-sistemas-y-servicios-de-sa lud\&Itemid=307 (acessado em 12/Ago/2018).

44. Pérez SL, Arrivillaga M. Redes integradas de servicios de salud en el marco de la atención primaria en salud en países seleccionados de América Latina. Salutem Scientia Spiritus (En línea) 2017; 3:32-49.

45. Ocampo M, Betancourt V, Montoya J, Bautista D. Sistemas y modelos de salud, su incidencia en las redes integradas de servicios de salud. Rev Geren Polit Salud 2013; 12:114-29.

46. Dirección de Presupuestos, Ministerio de Hacienda. Resumen ejecutivo elaborado por el panel evaluador e informe de comentarios a los resultados de la evaluación elaborado por la institución responsable del programa. https:// www.dipres.gob.cl/597/articles-139781_r_ ejecutivo_institucional.pdf (acessado em 12/ Out/2019).
47. Ministerio de Salud. DFL-1 2005 (art. 136). Fija texto difundido, coordinado y sistematizado del decreto Ley n. ${ }^{\circ} 2.763$, de 1879 , y de las leyes n. ${ }^{\circ} 18.933$ y n. ${ }^{\circ} 18.469$. https://www. leychile.cl/Navegar?idNorma $=249177$ (acessado em 29/Out/2019).

48. Bernales M, Cabieses B, McIntyre AM, Chepo M. Desafíos de la atención sanitaria de migrantes internacionales em Chile. Rev Peru Med Exp Salud Pública 2017; 34:167-75.

49. Horst B. Fuentes de financiamiento de gobiernos subnacionales y descentralización fiscal. Serie Informe Económico 2010; (202). https://archivos.lyd.org/other/files_mf/SIE202-Fuentes-de-Financiamiento-de-Gobier nos-Subnacionales-y-Descentralizacion-Fis cal-BHorst-En.pdf.

50. Vande N, Xu K, Soucat A, Fleisher L, Aranguren $\mathrm{M}$, Wang $\mathrm{H}$. Measuring primary health care expenditure in low-income and lower middle-income countries. BMJ Glob Health 2019; 4:e001497.

51. Fundación Equidad. Mesa social por el derecho a la salud promueve la idea de crear un sistema único y universal. https://equidad.cl/ index.php/noticias/312-mesa-social-por-elderecho-a-la-salud-promueve-idea-de-crearun-sistema-único-y-universal.html (acessado em 09/Dez/2019).

52. Parada M, Bass del Campo C. ¿Sistema, seguro o plan único de salud? Diario Uchile 2019; 13 dez. https://radio.uchile.cl/2019/12/13/siste ma-seguro-o-plan-unico-de-salud/.

53. Radio Cooperativa. Académicos del área de la salud piden un seguro público, universal y solidario. Cooperativa.cl 2019; 29 nov. https://www.cooperativa.cl/noticias/ pais/salud/fonasa/academicos-del-area-dela-salud-piden-un-seguro-publico-univer sal-y/2019-11-29/181838.html.

54. Sanabria CAP, Coelho TCB, Cortés FAM. Trajetória da privatização do sistema de saúde chileno (1924-2005). Saúde Debate 2020; 44:541-55. 


\section{Abstract}

Since the Declaration of Alma-Ata in 1978, primary healthcare (PHC) is considered an essential component of health systems. In the Chilean case, management of primary care was municipalized during the dictatorship and maintained by the subsequent governments, with some reforms. The aim of this article was to estimate and analyze spending in PHC in Chile, during the governments of Sebastián Piñera and Michelle Bachelet. Collection of financial data was oriented by the model of National Health Accounts (CNS), and later the amounts were deflated according to the Consumer Price Index. The principal source of information was the National System of Municipal Information (SINIM). The results show that during the period there was a permanent increase in spending in PHC; however, the average percent change was slightly higher in the first government compared to the second. The percentage of spending in PHC in relation to public spending in health was $21.4 \%$ for the eight years, with few variations. Indicators show that inequalities between administrative and health regions are increasing steadily. Therefore, although transfers to fund primary care services are increasing, they may be poorly distributed. This and other problems like the commodification of services and dismantlement of the network compromise the consolidation of PHC, especially in a health system based on contributive insurance like the Chilean system.

Healthcare Financing; Primary Health Care; Health Expenditures

\section{Resumen}

Desde la Declaración de Alma-Ata, en 1978, la atención primaria en salud (APS) está considerada un componente esencial de los sistemas de salud. En el caso chileno, la gestión de la atención primaria fue municipalizada durante la dictadura, y mantenida por los gobiernos posteriores con algunas reformas. El objetivo de este trabajo fue estimar y analizar el gasto en APS en Chile, durante los gobiernos de Sebastián Piñera y Michelle Bachelet. La recogida de datos financieros estuvo orientada por el Modelo de Cuentas Nacionales en Salud (CNS) y, posteriormente, los valores fueron deflactados según el Índice de Precios al Consumidor (IPC). La principal fuente de información fue el Sistema Nacional de Información Municipal (SINIM). Los resultados muestran que durante el período hubo un aumento permanente del gasto en APS; no obstante, la media de variación porcentual fue un poco mayor en el primer gobierno que en el segundo. El porcentaje del gasto en APS, en relación con el gasto público en salud fue de un $21,4 \%$ para los ocho años, teniendo pocas variaciones. Los indicadores muestran que la desigualdad entre las regiones administrativas y de salud está ampliándose progresivamente. Por ello, los fondos destinados a financiar los servicios ofrecidos en atención primaria, aunque crecientes, posiblemente están siendo mal distribuidos. Todo ello, junto con otros problemas, como la mercantilización de los servicios y la desintegración de la red, perjudica la consolidación de la APS, sobre todo si se trata de un sistema de salud basado en seguros contributivos como el chileno.

Financiación de la Atención de la Salud; Atención Primaria de Salud; Gastos en Salud
Recebido em 05/Jan/2020

Versão final reapresentada em 14/Jul/2020

Aprovado em 05/Ago/2020 